\title{
High energy and average power femtosecond laser for driving mid-infrared optical parametric amplifiers
}

\author{
P. Malevich, ${ }^{1}$ G. Andriukaitis, ${ }^{1}$ T. Flöry, ${ }^{1}$ A. J. Verhoef, ${ }^{1}$ A. Fernández, ${ }^{1}$ S. Ališauskas, ${ }^{1}$ \\ A. Pugžlys, ${ }^{1, *}$ A. Baltuška, ${ }^{1}$ L. H. Tan, ${ }^{2,3}$ C. F. Chua, ${ }^{4}$ and P. B. Phua ${ }^{2,3,4}$ \\ ${ }^{1}$ Photonics Institute, Vienna University of Technology, Gusshausstrasse 27-387, Vienna A-1040, Austria \\ ${ }^{2}$ Nanyang Technological University, 21 Nanyang Link, Singapore 637371 \\ ${ }^{3}$ DSO National Laboratories, 20 Science Park Drive Singapore 118230 \\ ${ }^{4}$ Temasek Laboratories, Nanyang Technology University, 50 Nanyang Drive, Singapore 637553 \\ ${ }^{*}$ Corresponding author: pugzlys@tuwien.ac.at
}

Received May 28, 2013; accepted June 21, 2013;

posted July 1, 2013 (Doc. ID 191198); published July 25, 2013

\begin{abstract}
We have developed the first (to our knowledge) femtosecond Tm-fiber-laser-pumped Ho:YAG room-temperature chirped pulse amplifier system delivering scalable multimillijoule, multikilohertz pulses with a bandwidth exceeding $12 \mathrm{~nm}$ and average power of $15 \mathrm{~W}$. The recompressed $530 \mathrm{fs}$ pulses are suitable for broadband white light generation in transparent solids, which makes the developed source ideal for both pumping and seeding optical parametric amplifiers operating in the mid-IR spectral range. (c) 2013 Optical Society of America

OCIS codes: (140.7090) Ultrafast lasers; (190.4970) Parametric oscillators and amplifiers; (140.3070) Infrared and far-infrared lasers.

http://dx.doi.org/10.1364/OL.38.002746
\end{abstract}

Ultrashort multimillijoule pulses in the eye-safe $2.1 \mu \mathrm{m}$ region are in demand for material processing, remote sensing, and spectroscopy [1] as well as for medical [2] and defense [3] applications. Pump lasers operating above the wavelength of $2 \mu \mathrm{m}$ are also required for efficient parametric generation of broadband femtosecond mid-IR pulses in zinc germanium phosphate (ZGP) crystals in the $4-10 \mu \mathrm{m}$ range $[4,5]$.

The gain bandwidth of $\mathrm{Ho}^{3+}$-doped crystals is sufficiently broad to support femtosecond operation. $\mathrm{Ho}^{3+}$, when doped into a YAG host, has its maximum emission cross section in the vicinity of $\sim 2.1 \mu \mathrm{m}$, which coincides with an atmospheric transparency window. It has a long upper state lifetime of $\sim 8 \mathrm{~ms}$, ensuring large energy storage and low saturation fluence of $\sim 6 \mathrm{~J} / \mathrm{cm}^{2}$, which allows efficient extraction of the stored energy. Therefore, laser crystals based on $\mathrm{Ho}^{3+}$ have great potential to operate in an amplifier-type configuration utilizing short pulse extraction [6].

The technology of Ho:YLF [7-9] and Ho:YAG [10-14] $Q$-switched laser systems generating multimilijoule narrowband nanosecond pulses is well established. $Q$-switching at subkilohertz repetition rates is mostly realized with acousto-optic modulators. Ho:YAG is typically pumped by Tm-doped fiber or Tm:YLF lasers, although recently direct laser diode pumping has also been reported [11]. However, despite a recently reported Cr-based amplifier delivering submillijoule femtosecond pulses at $2.4 \mu \mathrm{m} \mathrm{[15]} \mathrm{and} \mathrm{a} \mathrm{Ho:YLF} \mathrm{master} \mathrm{oscillator/RA}$ system generating picosecond $1.5 \mathrm{~mJ}$ pulses [16], to date there are no reports on the multimillijoule femtosecond pulse laser amplifiers operating around $2 \mu \mathrm{m}$.

One of the things impeding the development of $2 \mu \mathrm{m}$ femtosecond amplifiers is a lack of broadband modelocked oscillators operating in this spectral region. Only very recently femtosecond Tm: $\mathrm{Lu}_{2} \mathrm{O}_{3}$ [17] and Tm: $\mathrm{Sc}_{2} \mathrm{O}_{3}$ [18] oscillators operating, correspondingly, at 2076 and $2107 \mathrm{~nm}$ have been reported. However, the pulse energy of those Tm-based seeders-3.3 and 2.7 nJ respectively- is below the level required for achieving a high pulse contrast in a femtosecond Ho amplifier, as will be shown in this work.

In this Letter we present the first (to our knowledge) highly efficient femtosecond cw-pumped Ho:YAG chirped pulse amplifier (CPA) seeded by the output of an optical parametric amplifier (OPA). The system operates at room temperature and produces broadband pulses supporting an $\sim 440$ fs pulse duration at an energy level of up to $3 \mathrm{~mJ}$ at a $5 \mathrm{kHz}$ repetition rate, resulting in $15 \mathrm{~W}$ average power.

The system consists of the seed source and of the CPA, which includes the stretcher, the Ho:YAG regenerative amplifier (RA), and the compressor (Fig. 1). The RA is based on an antireflection coated, $0.5 \%$ doped $\varnothing 5 \times$ $50 \mathrm{~mm}$ Ho:YAG rod that is end-pumped by a $70 \mathrm{~W}$ Tm fiber laser (IPG Photonics). Due to the low damage threshold of Faraday rotators available for the wavelength of

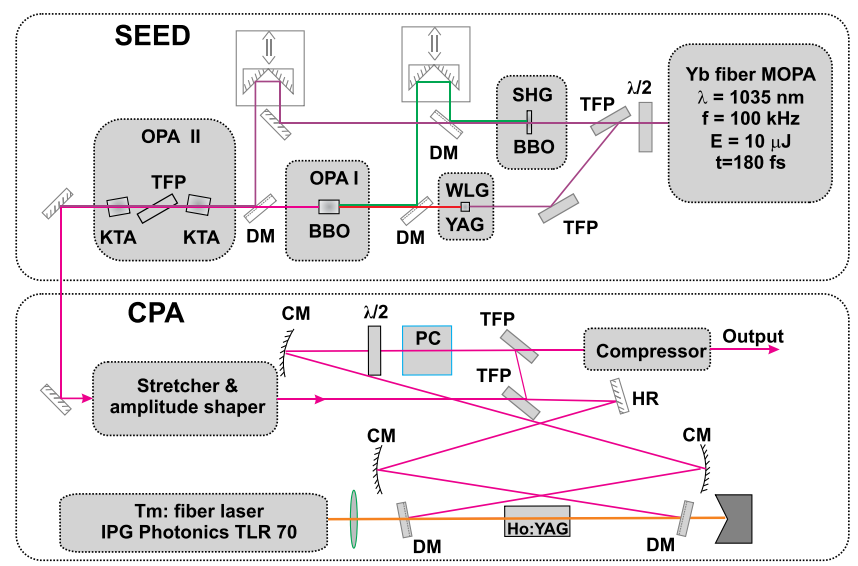

Fig. 1. Layout of the Yb-fiber master oscillator power amplifier (MOPA) pumped OPA seed source and Ho:YAG CPA system. TFP, thin film polarizer; $\lambda / 2$, half-wavelength retardation plate; WLG, white light generation unit; SHG, second harmonic generation unit; PC, Pockels cell; DM, dichroic mirror; CM, curved mirror; HR, high reflector. 
$2.1 \mu \mathrm{m}$, we have opted for a ring RA cavity design, in which the injection and output beam ports are separated and the amplification of the injected beam is unidirectional. Because of the ring-cavity design, the Pockels cell (rubidium titanyl phosphate, RTP; Raicol Crystals Ltd.) is operated in the half-wavelength regime. The length of the RA cavity is $4 \mathrm{~m}$, which corresponds to a round-trip time of $13.5 \mathrm{~ns}$.

In the cw mode, the Ho:YAG laser generates $28 \mathrm{~W}$ output power at an incident pump power of $68 \mathrm{~W}$, which corresponds to an optical-to-optical efficiency of $41 \%$. In the case of seeded operation, the RA is injected with $2.1 \mu \mathrm{m}$ pulses from a white light (WL) seeded two-stage OPA based on type I $\beta$-barium borate (BBO) and type II potassium titanyl arsenate (KTA) crystals (Fig 1). Two alternative sources-a femtosecond diode pumped solid state (DPSS) Yb:KGW amplifier (Pharos, Light Conversion) and a home-built $\mathrm{Yb}$ fiber amplifier producing $10 \mu \mathrm{J}, 180 \mathrm{fs}$ pulses centered at $1030 \mathrm{~nm}$ [19]-were successfully applied to pump the $2.1 \mu \mathrm{m}$ OPA. The repetition rate of $5 \mathrm{kHz}$ was chosen to maintain a reasonably high average output power while lowering the risk of voltageinduced darkening of the RTP Pockels crystal.

Because the generation of $2.1 \mu \mathrm{m}$ seed pulses via spectral self-broadening of $1030 \mathrm{~nm}$ pulses is challenging, we chose a cascaded OPA approach. A $1 \mu \mathrm{J}$ fraction of the $1030 \mathrm{~nm}$ output is focused into a $6 \mathrm{~mm}$ thick undoped YAG crystal for the generation of WL in the vicinity of $680 \mathrm{~nm}$. The remaining $1030 \mathrm{~nm}$ light is directed into $1 \mathrm{~mm}$ thick type I SHG BBO crystal for frequency doubling. The conversion efficiency was kept below $5 \%$ to avoid depletion of the $1030 \mathrm{~nm}$ pulses. The latter are subsequently separated by a dichroic mirror and are used to pump the second OPA stage. Amplification of the $680 \mathrm{~nm}$ WL in the type I BBO crystal (OPA I) pumped with $320 \mathrm{~nJ}$ $515 \mathrm{~nm}$ pulses generates idler pulses at $2.1 \mu \mathrm{m}$. To preclude angular chirp on the idler beam, the WL seed and $515 \mathrm{~nm}$ pump beams were overlapped strictly collinearly. The generated $2.1 \mu \mathrm{m}$ pulses are amplified in OPA II based on an inline pair of type II KTA crystals pumped at $1030 \mathrm{~nm}$. The second KTA crystal reverses the walk-off accumulated in the first KTA crystal and thus compensates spatial chirp on the amplified beam. To avoid interference caused by the group velocity mismatch between the $2.02 \mu \mathrm{m}$ signal and $2.1 \mu \mathrm{m}$ idler pulses, the $2.02 \mu \mathrm{m}$ pulses generated during the amplification of $2.1 \mu \mathrm{m}$ light in the first KTA crystal are filtered out by a thin film polarizer that is transparent for $1030 \mathrm{~nm}$ light.

The $2.1 \mu \mathrm{m}$ pulses with energy of $\sim 0.7 \mu \mathrm{J}$ and bandwidth of $40 \mathrm{~nm}$ FWHM (the spectrum is presented in the inset of Fig. 2) are stretched in a positive-dispersion Öffner scheme stretcher. The stretcher is based on a single $600 \mathrm{l} / \mathrm{mm}$ ruled diffraction grating and $R=-762 \mathrm{~mm}$ spherical mirror. The transmission of the stretcher is $\sim 22 \%$, which is determined by the efficiency of the diffraction grating and clipping of the spectral wings on the folding mirrors in the stretcher. The seed after the stretcher extends from 2080 to $2110 \mathrm{~nm}$ has an energy of $160 \mathrm{~nJ}$.

Spectral shaping of the seed pulses plays a crucial role in the presented CPA system. Without spectral shaping of the input pulses, the amplified spectrum after the RA collapses to a mere $2.4 \mathrm{~nm}$ FWHM centered at $2090 \mathrm{~nm}$. At higher pump levels, a weaker second band emerges at the

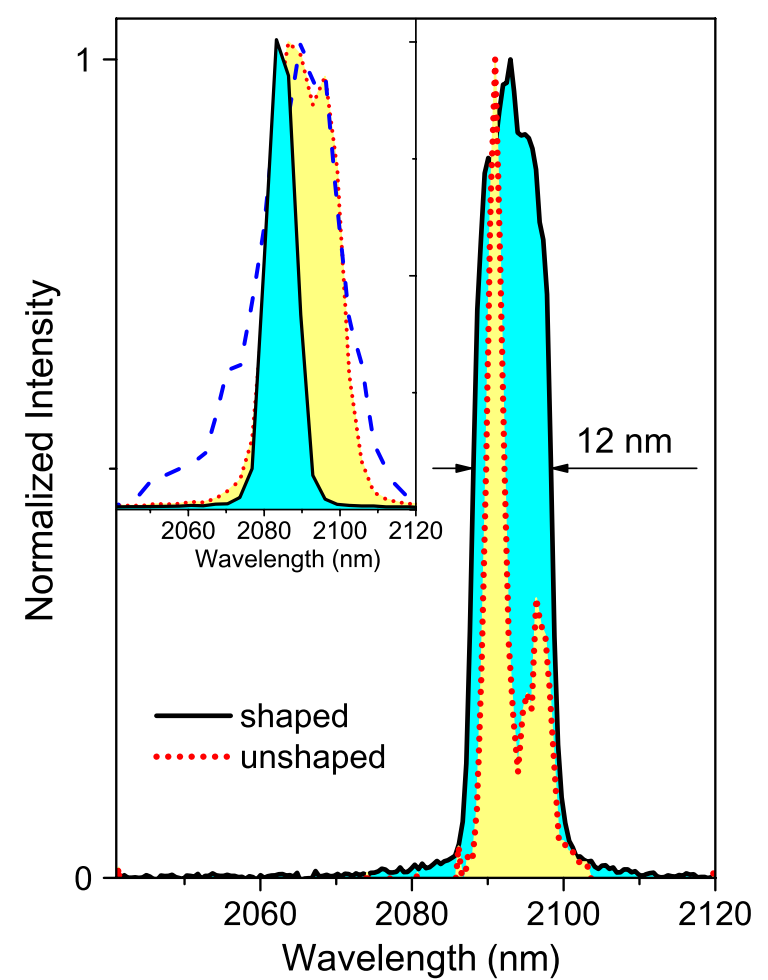

Fig. 2. Output spectra of the Ho:YAG RA for shaped (black solid line, cyan filled area) and unshaped (red dotted line, yellow filled area) seed. In the inset the output spectrum of the OPA (dashed blue line) and unshaped (red dotted line, yellow filled area) and shaped (black solid line, cyan filled area) spectra transmitted through the stretcher are presented. The fine structure of the shaped spectrum could not be resolved by our spectrometer (NIRQuest512).

$2097 \mathrm{~nm}$ gain peak of Ho:YAG (Fig. 2). To precompensate the gain narrowing in the RA cavity, we use a mechanical amplitude shaper located in the Fourier plane of the stretcher, which suppresses the intensity of the seed light in the spectral region where the gain is the highest. The shaping is primarily associated with the suppression of the red part of the seed spectrum, corresponding to the 2090 and $2097 \mathrm{~nm}$ gain peaks of Ho:YAG. Despite the additional loss of $60 \%$ of the seed pulse energy, the shaping results in a substantially broadened, $>12$ nm FWHM, amplified spectrum. Spectral shaping also helps to increase the stretching ratio of the seed pulses, which are measured by autocorrelation to be $>100 \mathrm{ps}$ in the shaped case and only $\sim 40 \mathrm{ps}$ in the absence of shaping. Correspondingly, the buildup of the nonlinear phase in the RA is reduced by spectral shaping.

The amplified pulses are recompressed in a negative dispersion compressor consisting of two $600 \mathrm{l} / \mathrm{mm}$ diffraction gratings separated by $120 \mathrm{~cm}$. The transmission of the compressor is $33 \%$ and is mainly determined by low diffraction efficiency of the gratings. The pulse duration obtained from the second harmonic generation frequency resolved optical gating (SHG FROG) measurements is $530 \mathrm{fs}$, whereas the Fourier transform limited pulse duration is $\sim 440 \mathrm{fs}$ (Fig. 3).

Before compression, when pumped with $55 \mathrm{~W}$ the amplifier generates $3 \mathrm{~mJ}$ pulses at a $5 \mathrm{kHz}$ repetition rate. The number of round trips in the RA was set to 22 . The change of the slope at high pump levels shown in Fig. $\underline{4}$ is 


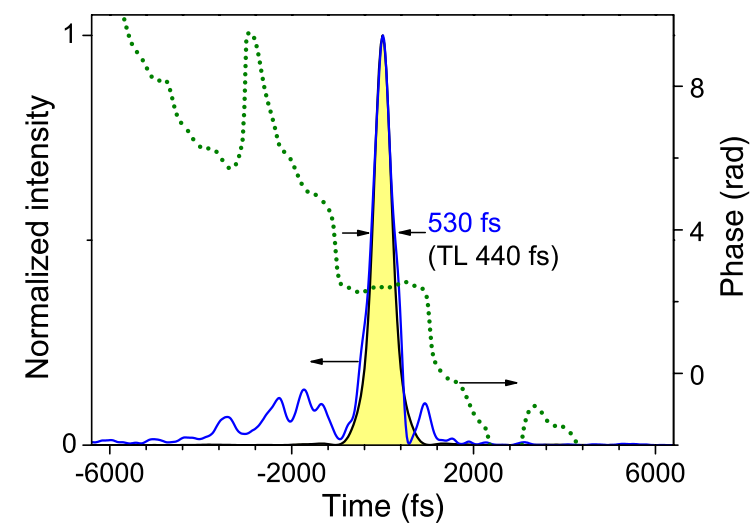

Fig. 3. Temporal profile and phase of compressed $2.1 \mu \mathrm{m}$ pulses as retrieved from SHG FROG measurements. The yellow-shaded contour shows the transform limited pulse.

due to a decreasing spatial overlap of the pump and laser mode volumes. Despite the fact that the cooling of the $50 \mathrm{~mm}$ long $\varnothing 5 \mathrm{~mm}$ Ho:YAG rod is done by simply running water through the holder, the polarized output of the RA exhibits an excellent spatial profile (inset in Fig. $\underline{4}$ ) with a beam quality factor $M^{2}=1.3$.

Because $Q$-switched radiation in a ring cavity develops in both directions, whereas the injected pulse is amplified only in one direction, the contrast of the amplified pulses with respect to the $Q$-switched background can be evaluated in a straightforward manner. The energy of the $Q$-switched background was determined by monitoring the energy leaking through one of the cavity mirrors (reflectivity 99.8\%) with a calibrated InGaAs photodiode.

These measurements - as presented in Fig. 5-show that the energy fraction retained by the $Q$-switche-d background is below $1 \%$ in the case when the seed energy exceeds $40 \mathrm{~nJ}$. Decreasing the seed energy worsens the contrast. At seed energy below $5 \mathrm{~nJ}$, more than $20 \%$ of the output energy is found in the nanosecond background. Because the spectral wings of the seed pulses (Fig. 2) are not amplified by the RA, the actual figures for the required seed energy level should be marginally lower than the ones given in Fig. 5. Therefore, the desired seed level for high-contrast amplification is in the range of several tens

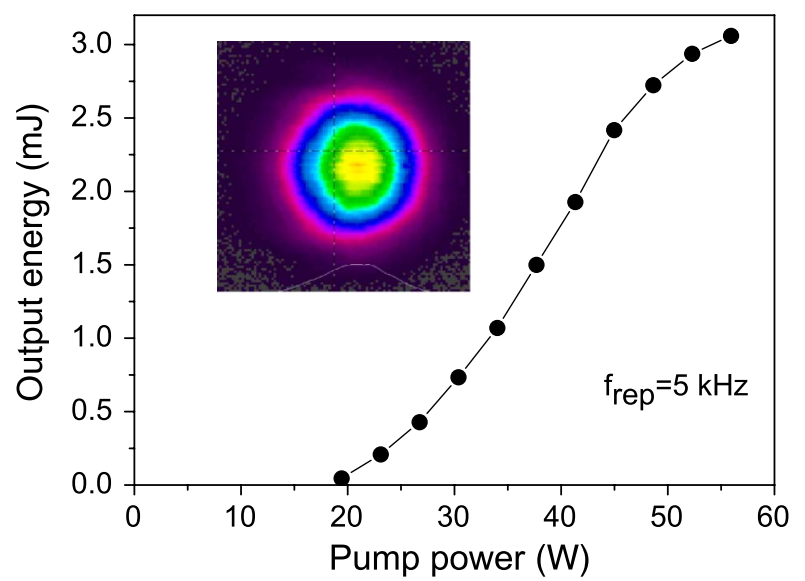

Fig. 4. Dependence of the output energy on pump power in the case of 22 round trips in the RA cavity and seed energy of $85 \mathrm{~nJ}$; in the inset, a far-field beam profile of the RA output is shown.

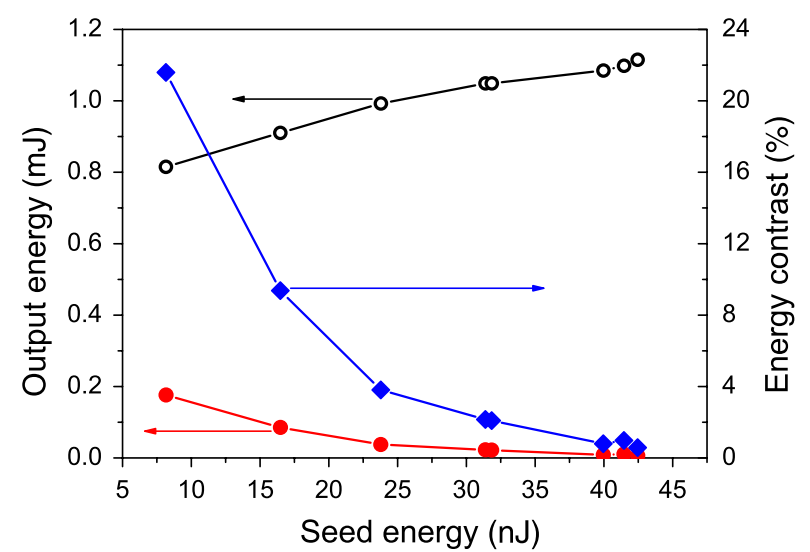

Fig. 5. Dependence of the output energy (left axis) contained in the amplified picosecond (open black circles) and $Q$-switched nanosecond (solid red circles) radiation on the seed energy; the solid blue diamonds show the dependence of the energy contrast (right axis) determined as the percentage of $Q$-switched nanosecond radiation with respect to the energy contained in the amplified picosecond radiation.

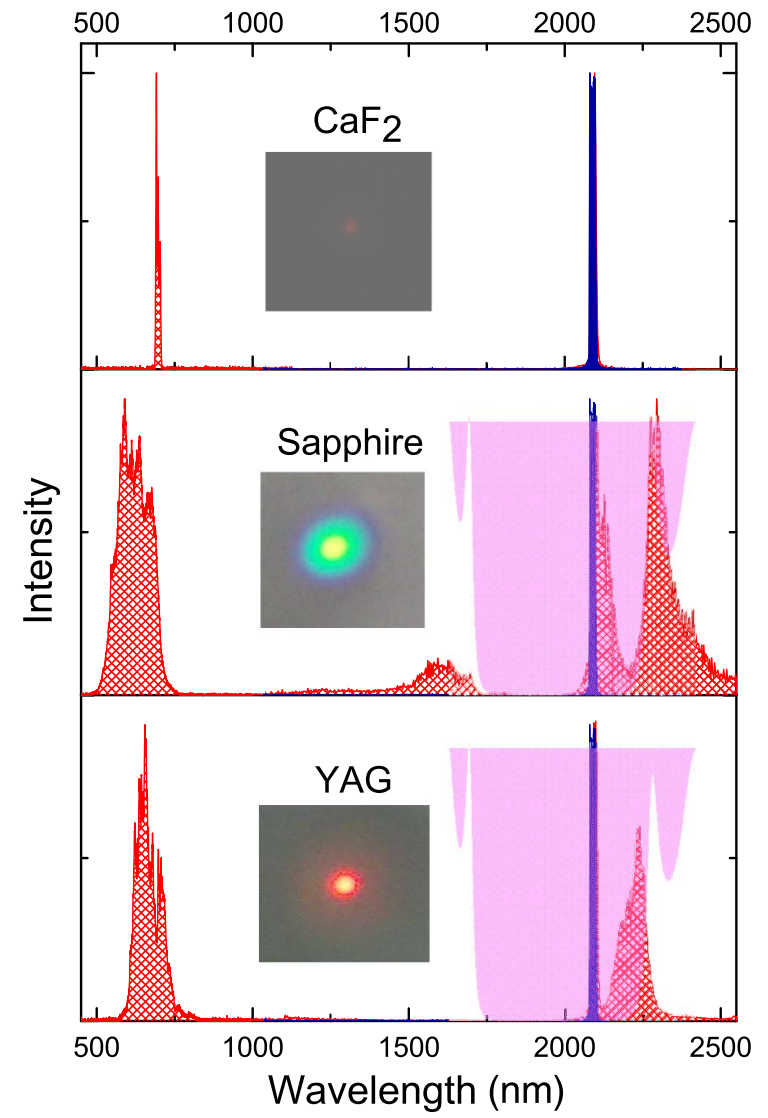

Fig. 6. Supercontinua (patterned red area) generated in different transparent solids (indicated in the panels). The shaded area is the transmission of the mirror cutting the fundamental $2.1 \mu \mathrm{m}$ light. The filled blue area represents the output spectrum of the Ho:YAG CPA system. In the insets, photographs of the generated supercontinua are presented. Spectra in the visible and IR spectral ranges, measured respectively with HR4000 and NIRQuest512 spectrometers (Ocean Optics), are independently normalized. The detection limit of our NIRQuest512 spectrometer lies at $2550 \mathrm{~nm}$. 
of nanojoules. Furthermore, with an increase in both the number of round trips and the pump power, a relative increase of the $Q$-switched background has been observed, which is attributed to a higher gain in the vicinity of $2090 \mathrm{~nm}$ as compared to the averaged gain that is experienced by the broadband preshaped picosecond pulses.

Preliminary experiments on WL generation in different transparent solids were performed with the compressed output of the Ho:YAG CPA (Fig. 6). Efficient spectral broadening in an $8 \mathrm{~mm}$ thick sapphire plate was observed in both the IR and visible spectral windows. The visible spectrum corresponds to the third harmonic of the broadened fundamental spectrum. Similar but less pronounced broadening was detected in a $6 \mathrm{~mm}$ thick undoped YAG crystal. By contrast, in $\mathrm{CaF}_{2}$ only the generation of the third harmonic was observed. These results provide further evidence on the importance of simultaneous spectral broadening around low-order harmonics in the process of supercontinuum generation with IR pulses [20]. As expected, filamentation and WL generation proceed only with femtosecond $2.1 \mu \mathrm{m}$ pulses and are immediately superseded by optical damage when the pulse compressor is detuned. The practical significance of the demonstrated spectral broadening is the ability not only to pump a mid-IR OPA but also to seed it with WL pulses at the signal wavelength.

In conclusion, we have demonstrated a first-of-its-kind (to our knowledge) high-intensity and high-averagepower femtosecond $2.1 \mu \mathrm{m}$ laser system that opens exciting opportunities in ablation-free surgery, multiphoton patterning of semiconductor crystals, nonlinear spectroscopy, efficient pumping of mid-IR few-cycle parametric amplifiers, and femtosecond filamentation. The system is operated with simple water cooling and is directly scalable in energy and average power.

This work has been supported by the Austrian Research Promotion Agency (FFG) via Eurostars Project 834722 (Mirandus), FP7 grant 244068 (Cross Trap), and Austrian Science Fund (FWF) grants F4903-N23 and I557-N16. A. Fernández acknowledges support from a Hertha Firnberg scholarship by FWF (grant T420-N16).

\section{References}

1. K. Scholle, S. Lamrini, P. Koopmann, and P. Fuhrberg, in Frontiers in Guided Wave Optics and Optoelectronics, B. Pal, ed. (Intech, 2010), pp. 471-500.
2. V. A. Serebryakov, E. V. Boiko, N. N. Petrishchev, and A. V. Yan, J. Opt. Technol. 77, 6 (2010).

3. G. Renz and W. Bohn, Proc. SPIE 6552, 655202 (2007).

4. V. Petrov, F. Rotermund, and F. Noack, J. Opt. A 3, R1 (2001).

5. G. Andriukaitis, S. Alisauskas, A. Pugzlys, A. Baltuska, L. H. Tan, J. H. W. Lim, P. B. Phua, K. Balskus, and A. Michailovas, in CLEO: Science and Innovations, 2012 OSA Technical Digest (Optical Society of America, 2012), paper CF3B.6.

6. S. A. Payne, L. L. Chase, L. K. Smith, W. L. Kway, and W. F. Krupke, IEEE J. Quantum Electron. 28, 2619 (1992).

7. A. Dergachev, D. Armstrong, A. Smith, T. Drake, and M. Dubois, Opt. Express 15, 14404 (2007).

8. W. Koen, C. Bollig, H. Strauss, M. Schellhorn, C. Jacobs, and M. J. D. Esser, Appl. Phys. B 99, 101 (2010).

9. H. J. Strauss, W. Koen, C. Bollig, M. J. D. Esser, C. Jacobs, O. J. P. Collett, and D. R. Preussler, Opt. Express 19, 13974 (2011).

10. P. A. Budni, C. R. Ibach, S. D. Setzler, E. J. Gustafson, R. T. Castro, and E. P. Chicklis, Opt. Lett. 28, 1016 (2003).

11. S. Lamrini, P. Koopmann, M. Schäfer, K. Scholle, and P. Fuhrberg, Opt. Lett. 37, 515 (2012).

12. G. Renz, P. Mahnke, J. Speiser, and A. Giesen, in Advanced Solid-State Photonics, 2011 OSA Technical Digest (Optical Society of America, 2011), paper AWA24.

13. Y.-J. Shen, B.-Q. Yao, X.-M. Duan, T.-Y. Dai, Y.-L. Ju, and Y.-Z. Wang, Appl. Opt. 51, 7887 (2012).

14. S. So, J. I. Mackenziea, D. P. Shepherd, and W. A. Clarkson, Proc. SPIE 6871, 68710R (2008).

15. E. Slobodchikov and P. F. Moulton, in CLEO: Applications and Technology, 2011 OSA Technical Digest (Optical Society of America, 2011), paper PDPA10.

16. A. Dergachev, presented at SPIE Photonics West, San Francisco, CA, February 2-7, 2013.

17. A. A. Lagatsky, O. L. Antipov, and W. Sibbett, Opt. Express 20, 19349 (2012).

18. A. A. Lagatsky, P. Koopmann, P. Fuhrberg, G. Huber, C. T. A. Brown, and W. Sibbett, in CLEO: Science and Innovations, 2012 OSA Technical Digest (Optical Society of America, 2012), paper CM1B.5.

19. A. Fernandez, L. Zhu, A. J. Verhoef, D. Sidorov-Biryukov, A. Pugzlys, A. Galvanauskas, F. O. Ilday, and A. Baltuška, Laser Phys. 21, 1329 (2011).

20. D. Kartashov, S. Ališauskas, A. Pugžlys, A. Voronin, A. Zheltikov, M. Petrarca, P. Béjot, J. Kasparian, J.-P. Wolf, and A. Baltuška, Opt. Lett. 37, 3456 (2012). 